\title{
HISTOLOGICAL AND IMMUNOHISTOCHEMICAL STUDY OF SELENIUM REGENERATIVE EFFECT ON SUBMANDIBULAR AND SUBLINGUAL GLANDS OF AGING RATS
}

\author{
Heba A. Elsaied*
}

\begin{abstract}
Background: The role of Selenium as an antioxidant has become a point of interest for its importance in prevention of cancer and other systemic diseases especially those associated with aging.

Objectives: The aim of the present investigation was to investigate the effect of Selenium supplementation on the submandibular and sublingual salivary glands of aging rats through:

1- Histological examination.

2- Immunohistochemical localization of Ki67.
\end{abstract}

Materials and Methods: Thirty young adult male albino rats with body weight ranged from 80110 gram and of 2 month age were used and divided into the following groups. Group I (control group): Consisted of 20 rats, subdivided into 2 subgroups. Group I.1:10 animals were sacrificed after one month to serve as young controls. Group I.2:10 animals were left till the end of the experiment to serve as old controls. Group II: 10 animals received Selenium $150 \mu \mathrm{g} / \mathrm{kg}$ diet for the whole experimental period. The experiment lasted for 9 months.

Results: histological results revealed that aging caused devastating effects on the histological structure of submandibular and sublingual salivary glands. Selenium markedly modulated the action of aging process and increased the regenerative capacity of submandibular and sublingual glands. According to expression intensity of Ki67 stain in submandibular and sublingual glands, there was statistically significant difference between young, selenium and old groups. In general, expression intensity of Ki67 stain was the highest in the young control group followed by selenium group and lowest in old control group. Also, ki67 expression was significantly higher in sublingual than submandibular glands.

Conclusions: Selenium was documented to have a powerful and beneficial antiaging effect. This faced lights around the promising role of selenium in the new era of regenerative medicine. Sublingual gland was more resistant to regressive changes of aging than submandibular gland.

KEY WORDS: Aging, Selenium, Submandibular Gland, Sublingual gland, Ki67.

\footnotetext{
* Lecturer in Oral Biology Department, Faculty of Dentistry, Suez Canal University.
} 


\section{INTRODUCTION}

One of the characteristics of aging is the progressive loss of physiological integrity, which results in the impairment of function and the increase in vulnerability to death. Such deterioration is considered the key factor of risk for major human pathologies including cancer, diabetes, cardiovascular disorders, and neurodegenerative diseases. ${ }^{(1)}$

There has been an outstanding advancement over recent years in research into aging, especially after discovering the possibility of controlling aging, at least to some extent, by employing genetic pathways and biochemical processes.

The common denominations of aging in various organisms are represented by nine tentative hallmarks with specific focus on aging in mammals. These hallmarks are: genomic instability, telomere attrition, epigenetic alterations, loss of proteostasis, deregulated nutrient sensing, mitochondrial dysfunction, cellular senescence, stem cell exhaustion, and altered intercellular communication. The ultimate objective is the identification of pharmaceutical targets in order that the human health could be improved during aging with the lowest level of side effects. ${ }^{[1]}$

There is a relationship between the degradation of salivary glands and radiation therapy, Sjögren's syndrome and/or aging. In addition, such degradation is considered a key clinical problem resulting in decreasing the oral health and the overall health in many patients. Further, there is no effective treatment currently and therefore, there is an urgent need for methods that enable the regeneration of salivary glands and restoration of saliva secretion. ${ }^{[2]}$

The aging of stem cell can be prevented by Bmi-1 and this is, partially at least, achieved by preventing the expression of cyclin-dependent kinase inhibitor p16 $6^{\text {Ink4a }}$. Consequently, the key to the loss of tissue homeostasis and developing associated degenerative diseases during aging is the dysregulation of the Bmi-1/p16 $6^{\text {Ink4a }}$ pathway. This process includes the molecular mechanisms, which underlies the decline caused by aging of submandibular salivary gland function. In turn, this is considered a significant problem among old people. ${ }^{[3]}$

Through the observation of histological samples, a more even and compact lobar structure with uniform appearance of parenchymal elements was shown by the glands of a young individual compared to an older one. Despite the fact that there was no difference in the number of ducts in the salivary glands, there was an increase in the proportional volume of fat and fibrovascular tissue of the parotid and submandibular glands in aging individuals. On the contrary, there was decrease in the proportional volume of secretion of acinar cell in aging individuals and therefore, it was considered as a key reason for dry mouth. Further, all these histological changes can lead to the overall hypofunction of salivary gland. ${ }^{[4]}$

Free radicals are characterized by being "uncharged, very reactive, and short-lived molecules or molecular fragments that contain one or more electrons in atomic or molecular orbitals". For humans, they have10,000-20,000 free radicals that can attack each individual cell of the human body. The lack of control over the generation of free radicals in human body can cause several ill effects including autoimmune diseases, heart and neurodegenerative diseases, cardiovascular diseases, diabetes, ischemia-reperfusion injury and aging. ${ }^{[5]}$

However, the chemicals, which have the ability to bind with free radicals and void their effect so as not to damage the biological molecules, are the antioxidants. Human body performs the production of endogenous antioxidants and accordingly, they are employed to combat different free radicals. Unfortunately, the majority of antioxidants are obtained from external resources. In particular, diet is considered as exogenous source of antioxidants 
or dietary antioxidants. In addition, brightly colored vegetables, fruits, and grains are considered the major sources of such class of antioxidants. Berries, green tea, and dark chocolate are also considered very effective sources. Currently, there are several oral supplements in the market, which are labeled as dietary antioxidants. ${ }^{[6]}$

The chemical element that has the symbol "Se" and the atomic number 34 is Selenium, which is a nonmetal (more rarely considered a metalloid). In addition, from Ancient Greek (Selene) means "Moon", Selenium was discovered in 1817 by Jon's Jacob Berzelius, who noticed that it is similar to tellurium (named for the Earth) that was previously discovered. ${ }^{[7]}$

When used in large amounts, the Selenium salts are toxic. However, it is necessary to use them in trace amounts for cellular function in many organisms including all animals. Furthermore, the Selenium is one of the ingredients of many multivitamins and other dietary supplements and it is one of the components of antioxidant enzymes glutathione peroxidase and thioredoxin reductase (that can indirectly lead to reducing certain oxidized molecules in animals and some plants). ${ }^{[8]}$

Selenium supplementation was effective to reduce salivary glands damage by $\left({ }^{131} \mathrm{I}\right)$ radiation in patients with differentiated thyroid cancer. ${ }^{[9]}$

Non-pharmacological therapies' potential to improve xerostomia after radiotherapy such as using Chinese herbs or trace minerals (zinc or selenium) has been examined by several studies. ${ }^{[10]}$

Furthermore, it is found that oxidative stresses can result from the decrease in the level of selenium. In a recent study, patients with oral mucositis resulting from a high level of chemotherapy were effectively able to reduce the term and seriousness of oral mucositis through the sufficient reinforcement of selenium, which has the ability to produce cytoprotective impact and antiulcer activity ${ }^{[1]]}$.
Antigen Ki-67 is a protein suitable for labeling dividing human cells . During the interphase, the antigen can be found inside the cell nucleus. At the time of mitosis, much of the protein is shifted to the surface of the chromosome. The fact that the protein is present during the active $\mathrm{G}(1), \mathrm{S}, \mathrm{G}$ (2) and $\mathrm{M}$ phases of the cell cycle but is absent in the G (0) phase of quiescent cells, makes it one good marker to determine the growth fraction of a cell population. ${ }^{[12]}$

A study found that expression of Ki-67 sharply decreased with advancing age or life history stages in the mouse brain. ${ }^{[13]}$

In the present investigation, we concentrated on studying the promising role of selenium in reversing the degenerative effects of cellular senescence on submandibular and sublingual salivary glands which ensures this antioxidant capability in regaining the normal histological architecture and subsequently functions of these glands.

\section{Aim of the study:}

The aim of the present investigation was to evaluate the effect of Selenium supplementation on the submandibular and sublingual salivary glands of aging rats through:

\section{1- Histological examination.}

\section{2- Immunohistochemical localization of Ki67.}

\section{MATERIALS AND METHODS}

\section{Sample size calculation:}

Sample size calculation was performed using G*Power version 3.1.9.2, Faul et al., (2007), University Kiel, Germany. Copyright (c) 1992-2014 ${ }^{[14]}$.

The effect size was 0.78 using alpha $(\alpha)$ level of 0.05 and Beta $(\beta)$ level of 0.05 , i.e., power $=$ $95 \%$; the estimated sample size (n) was a total of $\mathbf{3 0}$ samples for three groups. 


\section{Grouping:}

Thirty young adult male albino rats with body weight ranged from 80-110 gram and of 2 month age were used and divided into the following groups:

Group I (control group): Consisted of 20 rats, subdivided into 2 subgroups.

- Group I.1:10 animals were sacrificed by cervical dislocation after one month to serve as young controls.

- Group I.2:10 animals were left till the end of the experiment to serve as old controls.

Group II (selenium group): 10 animals received Selenium $150 \mu \mathrm{g} / \mathrm{kg} \operatorname{diet}^{[15]}$ for the whole experimental period. The experiment lasted for 9 months.

Selenium was supplied in powder form (Sigma Chemical Company; Tanta, Egypt) that was measured using sensitive scale then mixed with diet.

The animals were caged, five animals per cage and labelled according to their group, they were maintained under optimum conditions of good ventilation and temperature. Animals were supplied natural diet consisting of coarse corn barley and powdered milk and drinking tap water adlibitum. The experimental work of this study was performed in the animal house of faculty of dentistry, Suez Canal University.

The percentage of mortality for each group were calculated and recorded (no mortality cases encountered). Gross examination of head and neck of each animal was carried out through the whole experimental period.

At the end of the experiment which lasted for one month for group I.1, 9 months for groups I.2 and II the animals of different groups were sacrificed by cervical dislocation. Their submandibular and sublingual salivary glands were dissected.

For the histological investigation, processing was carried out on half of the specimens of submandibular and sublingual salivary glands, and they were stained with hematoxylin and eosin (H\&E) stain in order to be structurally investigated to identify if there are any possible microscopic changes.

Using Immuno-peroxidase avidin/biotin staining, the other half of the specimens was stained for immunohistochemical localization of ki67 in the glandular tissue, which was incubated by primary monoclonal mouse anti-rat ki67 antibody, and the color was developed by DAB.

By substituting the primary antibody by a nonspecific serum of the same dilution as its respective antibody, negative controls were prepared for ki67 immunohistochemical localization, procedures were continued as usual.

From each submandibular and sublingual salivary glands stained with $\mathrm{H} \& \mathrm{E}$ and from those stained with Immuno-peroxidase avidin/biotin staining for immunohistochemical localization of ki67, sections were selected, examined and photographed with Leica DM 1000 light microscopy and Camera using Leica Application suite-LAS software in the Centre Of Excellence Of Molecular And Cellular Medicine (CEMCM), Suez Canal University.

For Ki-67, the nuclear (nucleoplasmic) staining was considered to be positive. Using the light microscopy, Ki-67-positive nuclei were evaluated on each slide. ${ }^{[16]}$ Through a minimum of 200 cells per field in five different fields, the Ki- 67-positive nuclei were counted and the Ki- 67 labeling index (Ki-67 LI= the number (\%) of positive cells) was then calculated ${ }^{[17]}$.

\section{Visual Scoring Analysis}

From counting 200 cells in five different fields of each slide at a magnification $\mathrm{x} 400$, the percentage of cells that were positively stained was obtained to determine Ki-67 labeling index (LI). For the analysis, the average of five counts was used as ki-67 LI. Regardless of the intensity, any nuclear 
staining was considered immunopositive for $\mathrm{Ki}-67^{[18]}$. The Ki-67 (LI) was expressed as a percentage of immunoreactive positive proliferative cells to the total counted resting cells.

Immunopositive labeled cells for ki67 were scored using visual counting for each slide of the young \& old control and selenium groups' sections (score $1=1-10 \%, 2=11-25 \%, 3=26-50 \%, 4=51-$ $75 \%, 5=76-100 \%)^{[19]}$.

\section{Digital Surface Image Analysis}

Using the image analysis software (Image J, $1.29 t$, NIH, USA), Immunoreactivity of Ki-67 was evaluated. By employing a high resolution Leica DM 1000 camera in regions with subjectively the highest number of immunoreactivity cells, Images were obtained. At the power of $200 \mathrm{x}$, totally four adjacent medium-power, microscopic fields were analyzed. ${ }^{[20]}$ Instead of operator-guided color thresholding, automatic color thresholding was adopted to achieve maximum standardization. Computerized calculation of the total surface area of immunoreaction was expressed as a fraction (percentage) of the total surface area of the microscopic field.

\section{RESULTS}

\section{A) Hematoxylin and Eosin results:}

\section{Group I (control group):}

\section{Group I.1 (young controls):}

Examination of the H\&E stained sections revealed submandibular salivary gland with normal architecture.

The gland was encapsulated by fibrous connective tissue capsule that sent out septa that divided the gland into lobes and lobules. The formation of the gland comprised mixed predominately serous acini, intercalated, granular convoluted tubules, striated and execratory ducts. The mucous part took the form of few terminal portions where it was difficult to localize them near the periphery of the gland. For the serous acini, it was nearly spherical in shape and comprised pyramidal cells surrounding a narrow lumen. In addition, cells were characterized by having granular, moderately basophilic cytoplasm and rounded basally situated nuclei, whereas the ducts were characterized by being more acidophilic. (Fig. 1.A).

For the granular convoluted tubules (GCTs), they were large and mostly rounded. In addition, they were lined with tall columnar cells with large rounded basely situated nuclei, and apical eosinophilic granules (Fig. 1.B). As for The Intercalated ducts, they hardly recognized due to being compressed between the acini. Further, they were small and had cuboidal cell lining, centrally situated nuclei and little cytoplasm (Fig. 1.A). Lined by columnar cells, the striated ducts had centrally placed nuclei and intensely eosinophilic cytoplasm with basal striations. The blood vessels have occasionally accompanied the ducts (Fig. 1.A).

The excretory ducts were detected in the connective tissue septa between the lobes. They were lined by pseudostratified columnar epithelium with goblet cells and showed empty lumen of various diameters (Fig. 1.C). All the above parenchymal elements were invested in the reticular connective tissue stroma consisting of collagen fibers, fibroblasts and other connective tissue cells embedded in the ground matrix, together with blood vessels, lymphatics and nerves.

Large pyramidal mucous cells having abundant pale blue vacuolated cytoplasm were contained in sublingual gland acini, and the flattened basal nuclei and pale eosinophilic cytoplasm were contained in the acinar mucous cells. It was characterized by having large excretory ducts and few striated ducts, and mucous acini was mainly included therein with sets of serous cells, which looked crescents at the edges of the mucous acini (crescents or demilunes of Gianuzzi or of Von Ebner) (fig. 2 A, B). 

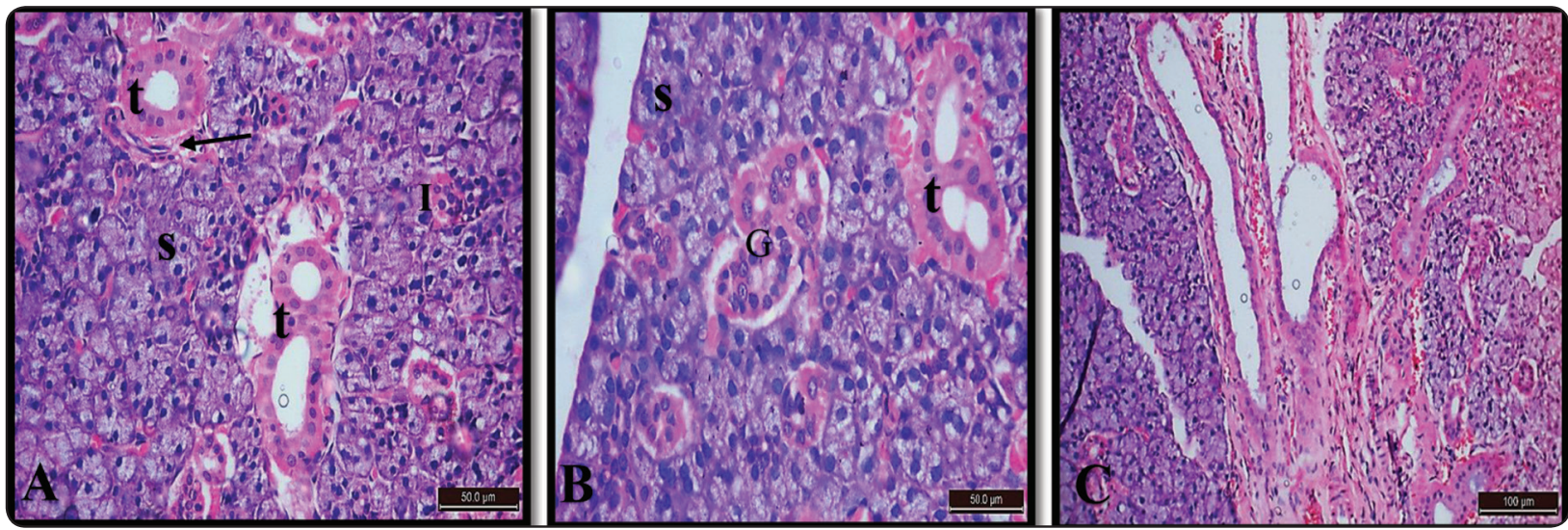

Fig. (1) Photomicrographs of Submandibular gland of rat from young control group showing normal gland architecture. (A) Serous acini with basophilic cytoplasm (s), striated ducts lined by columnar cells with basal striations (t). Intercalated duct lined by cuboidal cells (I). Some blood vessels appear in the connective tissue stroma around the ducts (arrows). (B) Serous acini, lined by pyramidal cells and basal rounded nuclei(s) and granular convoluted tubules (G) (Original magnification x 400). (C) Excretory duct with pseudostratified columnar lining and large empty lumen in interlober connective tissue septum. (Original magnification x 200) H\&E stain.

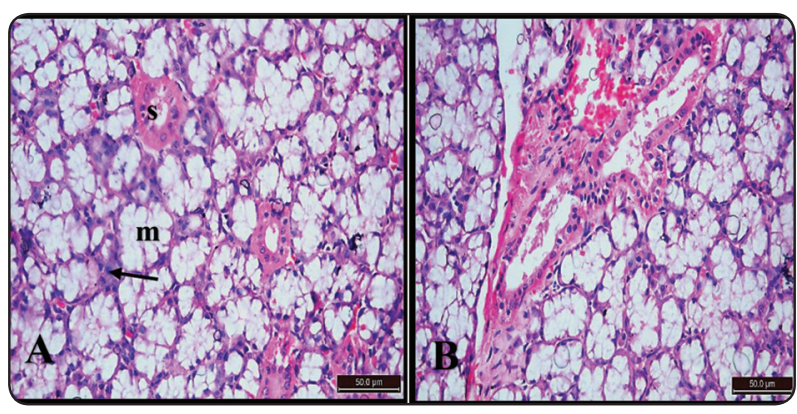

Fig. (2) Photomicrographs of Sublingual gland of rat from young control group showing (A) Acini contain large pyramidal mucous cells (m), striated ducts (s) and serous crescents or demilunes of Gianuzzi or of Von Ebner (arrow). (B) Excretory duct with pseudostratified columnar lining in interlober connective tissue septum. (Original magnification x 400) H\&E stain.

\section{Group I.2 (old controls):}

Examination of the H\&E stained sections of the submandibular salivary glands of diabetic rats revealed an apparent reduction in size and number of all the parenchymal elements including the acini and ducts in comparison to the controls. (Fig. 3.A)

The Serous acini appeared with a lot of cytoplasmic vacuolization. Some acini were lost where only few remnants were detected. The GCTs showed loss of regular configuration. The intercalated ducts showed marked shrinkage with cytoplasmic vacuolizations. The striated ducts were apparently reduced in size and some lining cells showed cytoplasmic vacuolizations and loss of basal striations. Neighboring blood vessels appeared dilated and congested with RBCs. (Fig. 3.B, and 3.C).

The excretory ducts possessed apparent reduction in the height of their cell lining with loss of their pseudo-stratification. Most of duct lumens were dilated and filled with pink stained homogenous stagnant secretion. Some of the ductal lining cells showed signs of degeneration manifested as shrinkage, loss of regularity and cytoplasmic vacuolizations. An increase in the fibrous C.T surrounding the ducts was seen. The blood vessels appeared dilated and congested with RBCs. (Fig 3.D).

Changes in the sublingual gland included widening in ducts lumens, secretion stagnation and slight blood vessels dilatation with their engorgement with red blood cells. Marked cellular inflammatory infiltrate replacing degenerated and atrophied parenchymal elements was encountered in very limited areas. (Fig. 4 A \& B) 


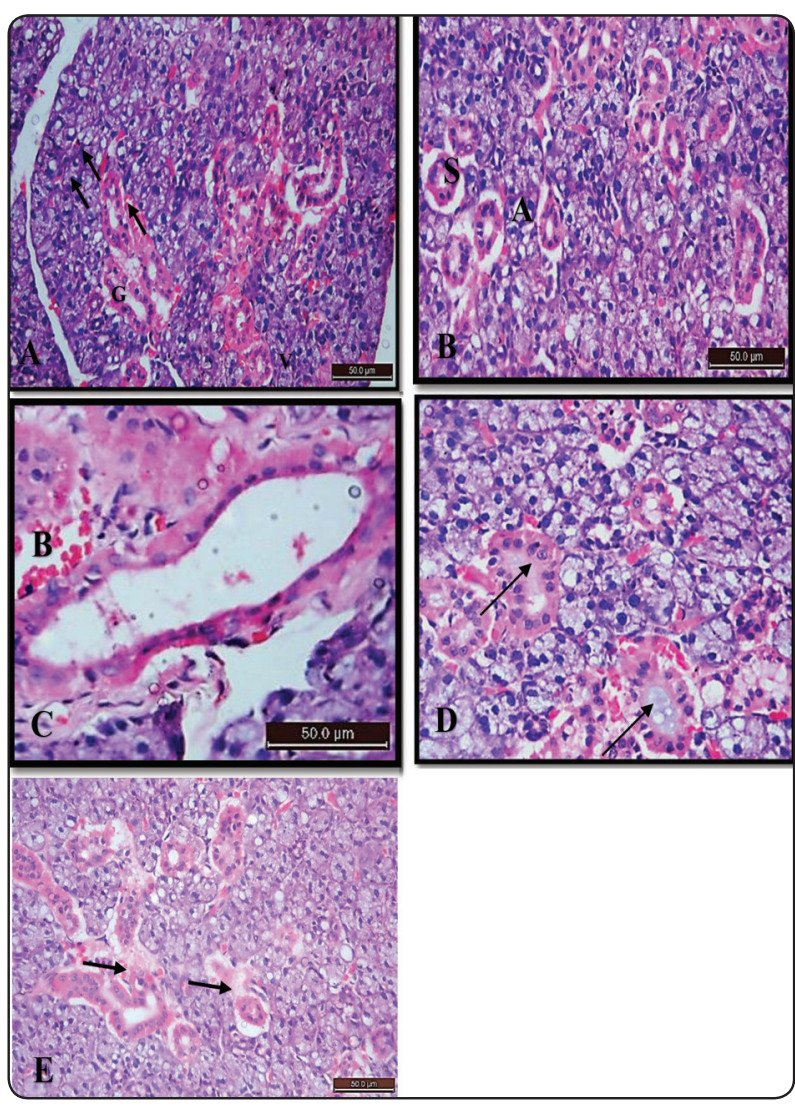

Fig. (3) Photomicrographs of submandibular gland of old control group showing (A) Serous acini with severe degrees of cytoplasmic vacuolization of the acinar cells (arrows). GCTs with loss of regular configuration $(\mathrm{G})$, striated ducts with cellular vacuolizations (V). (B) Serous acini with severe degrees of cytoplasmic vacuolization (A), shrinkage, atrophy and vacuolizations are also seen in striated duct (S). (C) Irregular lining of excretory duct with dilated blood vessels (B). (D) Stagnant secretion inside striated ducts lumen (arrows). (E) Replacement of some parenchymal elements with fibrous tissue (arrows). (Original magnification x 400) H\&E stain.

\section{Group II (selenium group):}

Examination of H\&E stained sections of submandibular salivary gland of rats received Selenium $150 \mu \mathrm{g} / \mathrm{kg}$ diet which lasted for 9 months showed improvements of their histological picture in comparison to those of the old control group. The gland represented almost normal histological picture. Acinar cells appeared with well-defined cell borders, basal rounded nuclei, and basophilic

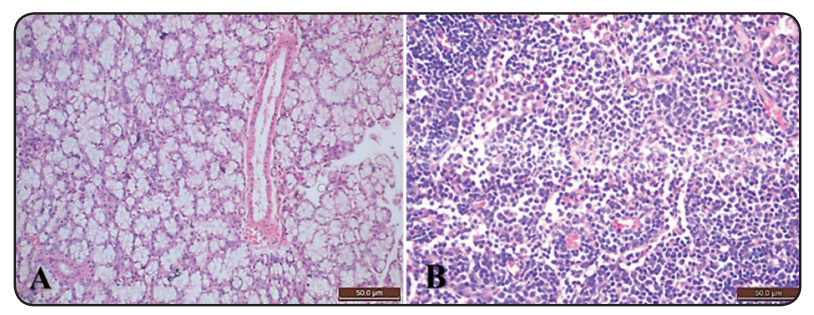

Fig. (4): Photomicrographs of sublingual gland of old control group showing (A) widening in duct lumen, secretion stagnation and blood vessels slight dilatation and their engorgement with red blood cells. (B) Marked cellular inflammatory infiltrate replacing degenerated and atrophied parenchymal elements in very limited areas. (Original magnification x 200) H\&E stain.

cytoplasm. Intra acinar cytoplasmic vacuolizations were occasionally found. Both granular convoluted tubules and striated ducts became almost normal. Normal blood vessels were detected while few others appeared dilated. (Fig. 5.A)

Sublingual gland showed almost normal mucous acini and striated ducts. Excretory ducts possessed cell lining with the pseudo-stratified columnar epithelial appearance. Most of duct lumens were normal or filled with small amount of secretion. (Fig. 5.B)

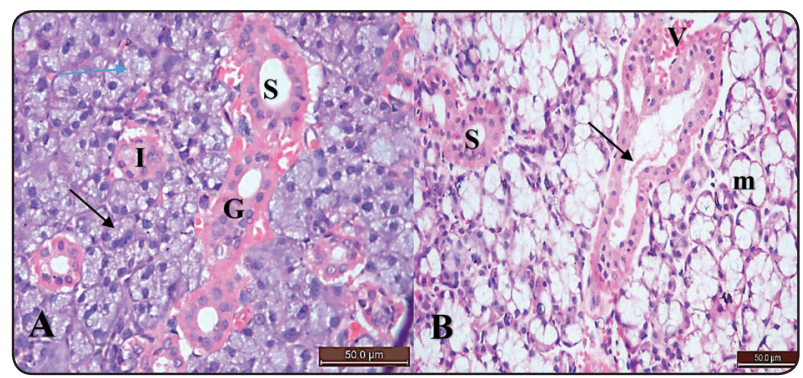

Fig. (5) A Photomicrographs of Submandibular gland of selenium group showing (A) Almost normal acini with normal basophilia and nuclei (arrow), striated duct with basal striations (S) and GCTs (G). Intercalated ducts with few intracytoplasmic vacuoles (I). (B) Photomicrograph of sublingual gland of selenium group showing almost normal mucous acini (m) and striated ducts (s). Regular lining of excretory duct with small amount of stagnant secretion inside lumen (arrow). Mildly dilated blood vessels (v). (Original magnification $\mathrm{x}$ 400) H\&E stain. 


\section{B) Immunohistochemical localization of Ki67}

\section{Negative control}

It was revealed by sections, which were taken from the submandibular and sublingual salivary glands of rats included in the young control group, incubated with non-specific serum and color developed by $\mathrm{DAB}$, that all the elements forming the gland had a negative staining reaction. (Fig. 6.A\& B).

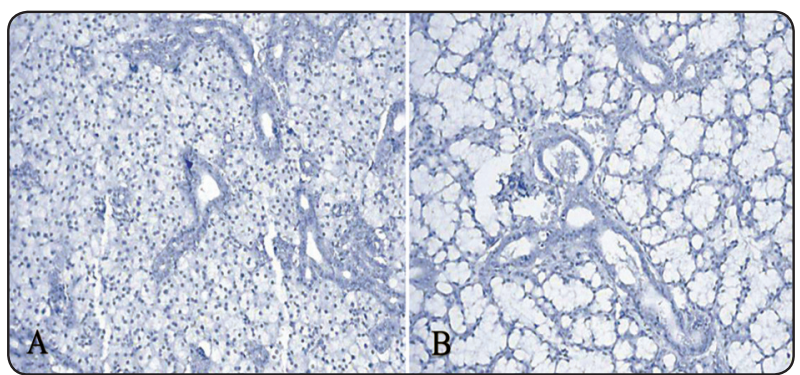

Fig. (6): The photomicrographs of Submandibular (A) and sublingual (B) glands of rats, which were included in the young control group, incubated with non-specific serum and color developed by $\mathrm{DAB}$, showed that all the elements forming the gland (Original magnification $\mathrm{x} 200$ ) had an negative staining reaction.

\section{Group I (control group):}

\section{Group I.1 (young controls):}

The submandibular and sublingual gland specimens taken from young control rats and incubated with primary monoclonal mouse anti-rat ki67 antibody and color developed by DAB revealed increased numbers of immunopositively labeled nuclear staining in the acini, striated and excretory ducts.

Numbers of immunopositively labeled score (2) (Fig. 7A) in serous acini and score (3) in mucous acini (Fig. 7B). The interstitial connective tissues have positive immunoreactivity.

\section{Group I.2 (old controls):}

The submandibular and sublingual gland specimens taken from old control rats and incubated with primary monoclonal mouse anti-rat ki67 antibody and color developed by DAB revealed decreased numbers of immunopositively labeled nuclear staining in the acini, striated and excretory ducts. The numbers of immunopositively labeled staining sections were with score (1) (Fig. 7C) in serous acini and score (2) in mucous acini (Fig. 7D).

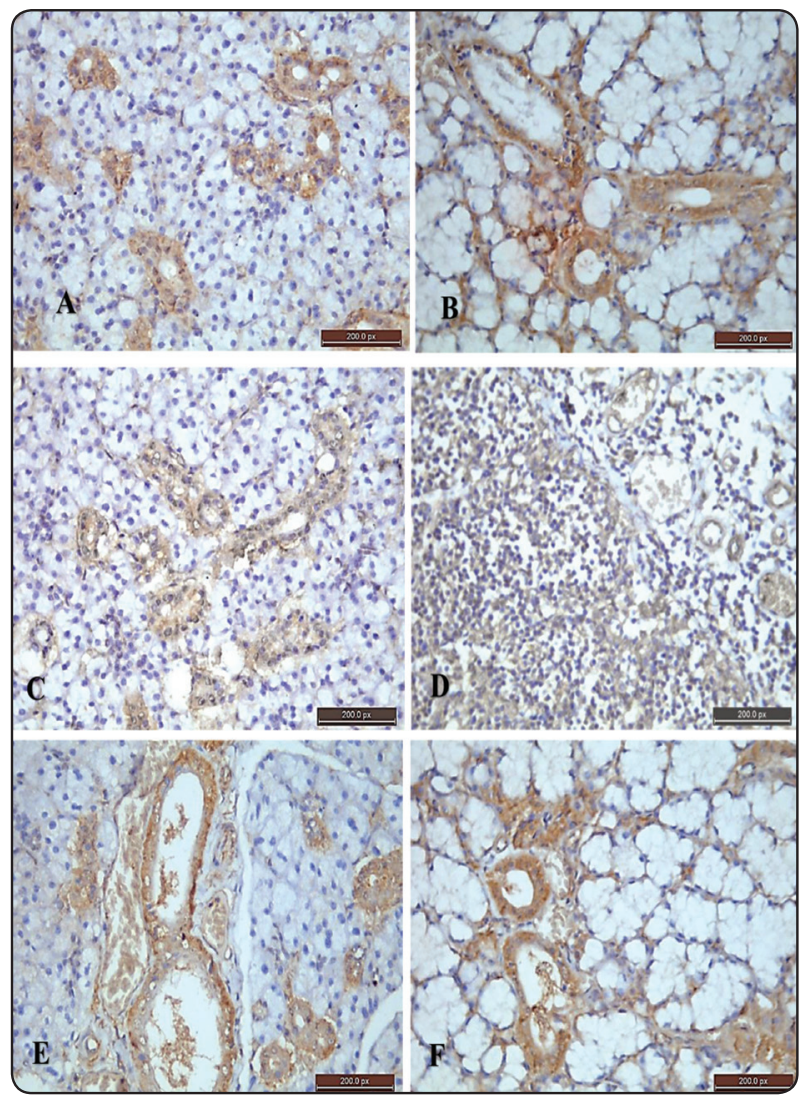

Fig.7: Photomicrographs of Submandibular (A) and sublingual (B) glands of rats from the young control group, incubated with ki67 monoclonal antibody showing immunopositively labeled cells score (2) (Fig. 7A) in serous acini and score (3) in mucous acini. Photomicrographs of submandibular (C) and sublingual (D) glands of rats from the old control group, showing the numbers of immunopositively labeled cells with score (1) in serous acini and score (2) in mucous acini.

\section{Group II (selenium group):}

The submandibular and sublingual gland specimens taken from rats received Selenium 150 $\mu \mathrm{g} / \mathrm{kg}$ diet for 9 months, incubated with primary monoclonal mouse anti-rat ki67 antibody and color developed by DAB revealed apparent increase 
in numbers of immunopositively labeled nuclear staining in the acini, striated and excretory ducts.

Abundant numbers of immunopositively labeled sections with score (2) (Fig. 7E) in serous acini and score (3) in mucous acini (Fig. 7F). The interstitial connective tissues have positive immunoreactivity.

Photomicrographs of Submandibular (E) and sublingual (F) glands of rats from the selenium group, showing abundant numbers of immunopositively labeled cells with score (2) in serous acini and score (3) in mucous acini. The interstitial connective tissues have positive immunoreactivity. (Original magnification x 400).

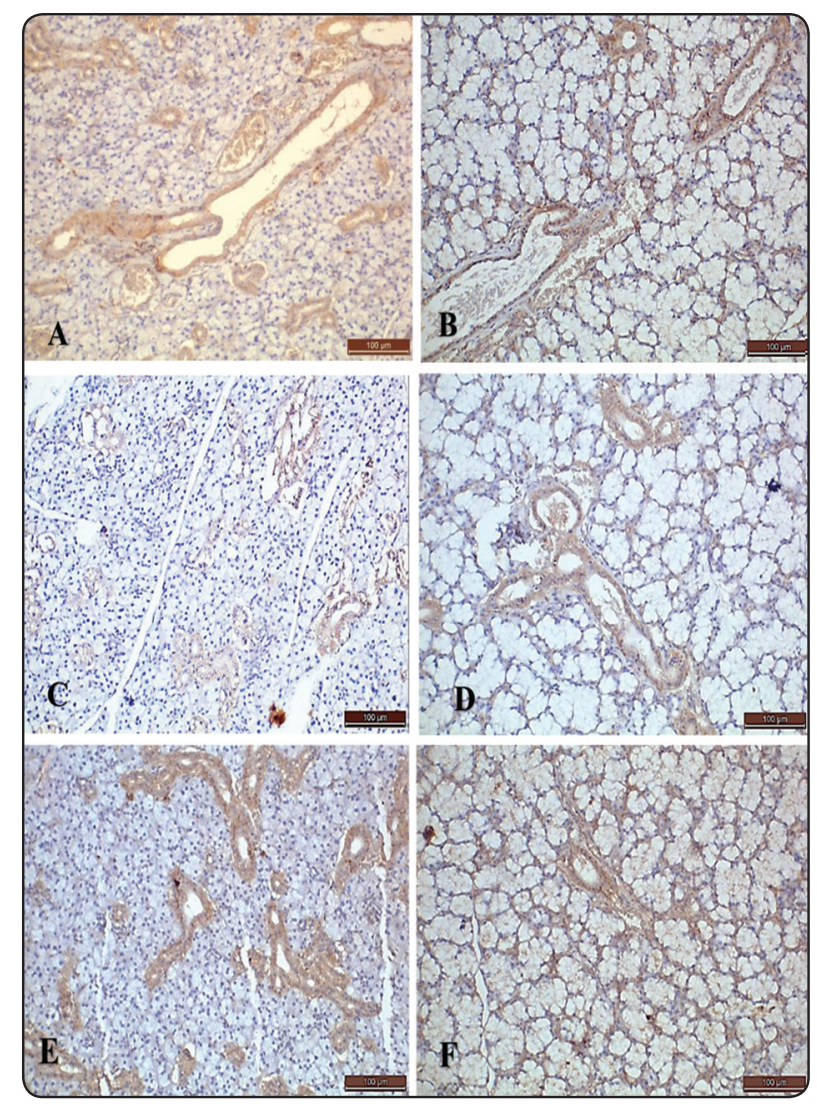

\section{Statistical analysis}

According to expression intensity of Ki67 stain in submandibular and sublingual glands the analysis of Variance test ${ }^{(21)}$ showed statistically significant difference between young, selenium and old groups in sublingual and submandibular gland with $P$-value $=0.016,0.001$ and 0.035 respectively. In general, expression intensity of Ki67 stain was the highest in young control then selenium groups, it recorded the lowest value for the old control group. According to Mann-Whitney test $(\mathbf{U})$, there was significant difference between sublingual and submandibular glands at young, selenium and old groups with $P$-value $=0.0001$ and 0.0003 respectively, when sublingual glands recorded higher value than its submandibular counterpart. Table (1), fig. (9).

Fig. (8): Photomicrographs of Submandibular (A) and sublingual (B) glands of rats from the young control group, showing strong immunopositive staining to ki67 antibody in the acini, striated and excretory ducts. Photomicrographs of submandibular (C) and sublingual (D) glands of rats from the old control group, showing weak immunopositive staining in the acini, striated and excretory ducts of submandibular gland, while sublingual gland shows weak staining in acini and moderate staining in striated and excretory ducts. Photomicrographs of Submandibular (E) and sublingual (F) glands of rats from the selenium group, incubated with ki67 monoclonal antibody showing moderate immunopositive nuclear staining in the acini, strong staining in striated and excretory ducts of submandibular gland. While the sublingual gland shows strong immunopositive nuclear staining in acini, striated and excretory ducts. (Original magnification $\mathrm{x} 200$ ). 
TABLE (1): Mean values for area of expression intensity of Ki67 stain in submandibular and sublingual glands of young control, selenium and old control groups.

\begin{tabular}{|l|l|l|l|l|}
\hline & Sublingual & Submandibular & Mann-Whitney test (U) & Sig. $^{2}$ \\
\hline Young Control & $194.86^{\mathrm{a}}$ & $184.80^{\mathrm{a}}$ & -2.419 & $0.016^{* *}$ \\
\hline Selenium & $183.62^{\mathrm{b}}$ & $175.19^{\mathrm{b}}$ & -3.25 & $0.001^{* *}$ \\
\hline Old control & $110.65^{\mathrm{c}}$ & $101.28^{\mathrm{c}}$ & -2.104 & $0.035^{* *}$ \\
\hline P values $<0.05$ & $0.0001^{* * *}$ & $0.0003^{* * *}$ & & \\
\hline
\end{tabular}

**, and different letters at the same column means significant difference between groups at $P$ - value $<0.05$.

*** Mann-Whitney $(U)$ test was used to compare between Sublingual and Submandibular glands (Row) at the same group at $P$ - value $<0.05$.

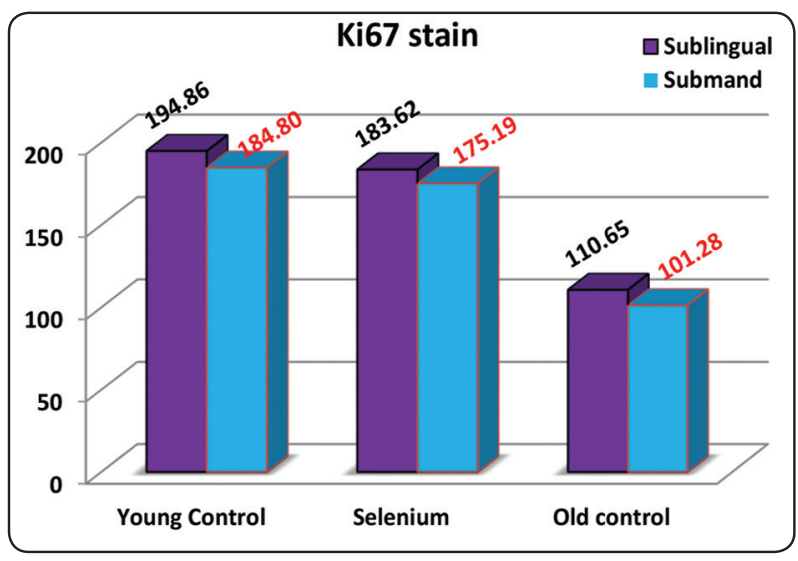

Fig (9): Double bar graph representing mean values for area of expression intensity of Ki67 stain in submandibular and sublingual glands of young control, selenium and old control groups.

\section{DISCUSSION}

Salivary glands degeneration and hyposalivation are considered noteworthy problems associated with aging where they are the reason for decreasing the oral health and overall health of many patients and currently there is no effective treatment for them. As a result, there is an urgent need for methods that can enable the regeneration of salivary glands and the restoration of saliva secretion.

In our current study, we focused on studying the antioxidant power of selenium in withdrawing the deteriorating effects of aging on submandibular and sublingual salivary glands and consequently salvaging functions of these glands.
In this investigation, examination of the $\mathrm{H} \& \mathrm{E}$ stained sections of the submandibular salivary glands of old control rats revealed an apparent reduction in size and number of all the parenchymal elements including the acini and ducts in comparison to the young controls.

The Serous acini, intercalated ducts and striated ducts appeared with a lot of cytoplasmic vacuolizations. Some of the ductal lining cells showed signs of degeneration manifested as shrinkage. The GCTs showed loss of regular configuration. Neighboring blood vessels appeared dilated and congested with RBCs. An increase in the fibrous C.T surrounding the ducts was seen.

Changes in the sublingual gland are limited to widening in ducts lumen, secretion stagnation and slight blood vessels dilatation with their engorgement with red blood cells.

Vacuolizations of acinar and ductal cells may be the result of edematous changes replacing degenerated or atrophic cells or may also be due to fatty degeneration of these cells.

Dilatation of blood vessels and their engorgement with RBCs which results in stasis and decrease in the blood flow are probably the possible causes of hypoxia and ischemia of the tissues that aggravates the degenerative effects of aging. 
Declined blood supply can lead to diminished oxygen and nutrient delivery to cells. This causes cell damage by producing toxic metabolites and reducing cellular energy. Also free radical generation especially reactive oxygen species (ROS) play a role in cell damage initiated by the reperfusion of blood flow to the ischemic organ ${ }^{[22]}$.

The histological results of current study are consisted with those of another study, which concluded that aging has general features including tissue dessiccation, reduced elasticity, diminished reparative capacity, altered cell permeability and increased calcium content in the cells of many organs. The ischemia generated by arteriosclerosis in periodontal tissues owing to the decrease in blood flow is hypothesized to be predisposing such tissues to fibrosis, loss of cellularity, and focal calcification ${ }^{[23]}$.

Moreover, the changes caused by aging in the submandibular glands (SMG) of male rats were examined morphometrically and ultra-structurally. Regressive changes of the granular ducts was the most conspicuous feature of the aged SMG. There was decrease in the extent and height of the granular ducts and the content of mature secretory granules. In addition, in the intercalated duct cells, cytoplasmic crystalloids, salivary deposits and oncocytic transformation were found therein ${ }^{[24]}$.

In the current study, changes in the sublingual gland included widening in ducts lumens, secretion stagnation and slight blood vessels dilatation with their engorgement with red blood cells in some areas. Marked cellular inflammatory infiltrate replacing degenerated and atrophied parenchymal elements was found in small areas.

These findings go with an investigation which found that several pathologies are associated with aging, such as atherosclerosis and inflammation ${ }^{[25]}$.

According to another study, in human sublingual glands, the aging process started with acinar atrophy, then duct-like structures existed and finally some parenchymal elements were replaced by fibrous and/or adipose tissue. The mononuclear infiltrate transformed from focal to diffuse ${ }^{[26]}$.

The results of an investigation revealed that acinar atrophy, duct-like structures, the distribution of mononuclear infiltrates, the replacement of parenchyma with fibrous or adipose tissue, and oncocytosis or mucous extravasation were the key histological changes taking place in the sublingual glands of edentulous patients with mouth floor enlargements, which were associated with aging ${ }^{[27]}$.

The mechanism of action of antioxidants is probably due to their ability to quench free radicals and protect against oxidative damage to DNA.

The essential trace element, whose low status in humans is linked to the increase in the risk of various diseases including cancer and heart disease, is Selenium (Se). Recently, there has been great interest in Se research due to the important role it plays in antioxidant selenoproteins to protect from oxidative stress initiated by excessive reactive oxygen species (ROS) and reactive nitrogen species $(\mathrm{NOS})^{[28]}$.

The acinar and ductal cells of submandibular and sublingual glands of the selenium group presented less atrophic changes with partial absence of intracytoplasmic vacuolizations. The cytoplasm was probably protected from the damaging effect of intermediate metabolic byproducts (free radicals) that were neutralized by antioxidant supplementation.

The beneficial role of selenium was obvious in the dietary management of chronic metabolic diseases such a Hyperlipidaemia, Hyperglycemia and Hyperphenylalaninemia ${ }^{[29]}$.

According to expression intensity of Ki67 stain in submandibular and sublingual glands the analysis of Variance test showed statistically significant difference between young, selenium and old groups 
in sublingual and submandibular gland with $P$-value $=0.016,0.001$ and 0.035 respectively . In general, expression intensity of Ki67 stain was the highest in young control then selenium groups, it recorded its lowest value for the old control group. According to Mann-Whitney test (U), there was significant difference between sublingual and submandibular glands at young, selenium and old groups with $P$-value $=0.0001$ and 0.0003 respectively, when sublingual glands recorded higher value than its submandibular counterpart.

There is a wide use of Ki67 staining as a proliferation indicator and its accumulation occurs only during S, G2, and M phases. Further, there is a continuous degradation of Ki67 in G1 and G0 phases, regardless of the reason for entry into G0/quiescence. As a result, during G0 and G1 in individual cells, its level is highly heterogeneous and dependent on how long an individual cell has spent in G0. Therefore, it is considered a graded rather than a binary marker for both the progression and time of cell cycle entry into quiescence ${ }^{[30]}$.

In the mouse hippocampus and subventricular zone of the aging mice, it was noticed that the number of nestin- and Ki-67-immunopositive cells decreased. ${ }^{[31]}$.

One of the most noticeable features of aging is the decrease in the regenerative potential of tissues ${ }^{[32]}$.

Moreover, an overall reduction in the activity of cell cycle of hematopoietic stem cells (HSCs), with old HSCs undergoing fewer cell divisions than young HSCs, was indicated by studies on aging in mice. There is a correlation between this and the accumulated DNA damage and the overexpressed inhibitory proteins of cell cycle such as p16INK4a ${ }^{[33]}$.

From our point of view, the existence of transforming growth factor $\beta$ (TGF- $\beta$ ), which has a significant role in mediating apoptotic cell death, might be the reason for the apoptosis of secretory and ductal cells in submandibular and sublingual salivary glands of aging albino rats.

Consequently, as a proof, treatment with TGFbeta inhibitor stimulated beta cell regeneration in both adult mice and humans' pancreas. The increase in beta cell proliferation was accompanied by a reduction in p16INK4a (cell-cycle inhibitory protein) levels and an increase in ki67 (proliferation indicator) expression in islets ${ }^{[34]}$.

This harmful and degenerative affection of aging on the salivary glands acini and ducts which consequently leads to alterations in their function (secretion of saliva) and precipitation of a condition of dry mouth that in turn will passively affect the integrity and health of oral structures including the salivary glands themselves, might be a possible reason for the age-related degeneration of theses glands.

A study demonstrated a significant increase of 3.2 times in the number of basal keratinocytes expressing Ki67, which is responsible of epithelium proliferation and thickness, after sodium selenite (NaSe) treatment. This provided a clarification of the key role played by Selenium in the replicative life span of keratinocytes and on aging skin. In addition, using Selenium in well-measured doses, can present a new strategy to protect skin against aging ${ }^{[35]}$.

The entry into $\mathrm{S}$ phase was slowed by the depletion of Ki-67 in human primary fibroblast cells and it also led to down-regulating of genes related to DNA replication ${ }^{[36]}$.

A study was made to evaluate histomorphometric age-related changes in labial salivary glands (LSG) of healthy individuals, with special reference to the mucous and seromucous acini. It concluded that there was marked decrease in the mean volume fraction $\left(V_{\mathrm{v}}\right)$ of seromucous acinar cells accompanied by a lower decrease in the $V_{\mathrm{v}}$ of mucous acinar cells ${ }^{[37]}$. These findings correlates with those 
of our study as ki67 expression was significantly higher in sublingual than submandibular glands. This enlightens the relative resistance of sublingual salivary gland to regressive changes more than submandibular gland.

Finally, it is important to note that Selenium can promote the progression of cell cycle and prevent cell death at nutritional doses. On the contrary, when does are supra nutritional, i.e. they are greater than the nutritional requirement but not toxic, Selenium can induce arrest of cell cycle and apoptosis. The key mechanism, by which Se exerts its biological functions including anticarcinogenic activity, protection against oxidant damage or aging, and even a role in reproduction and detoxicity, is the Se-modulation of cell cycle and apoptosis. More important, through the chemical forms and doses of Se, its molecular targets and the effectiveness of action are dictated ${ }^{[38]}$.

\section{REFERENCES}

1. Carlos L, Maria A, Linda P, Manuel S and Guido K. The Hallmarks of Aging. Cell 2013; 153:1194-1217.

2. Kihoon N, Deana C, Browna R, Smith J, Pedro L and Andreadisbcd O.Synergistic Effects of Laminin-1 Peptides, VEGF and FGF9 on Salivary Gland Regeneration. Acta Biomaterialia. Available online 25 April 2019. In Press, Accepted Manuscript.

3. Kimi Y, Satoshi K, Mayu I, Hiromi K, Atsushi O, Madoka I, Naoko O, Eiji H and Mitsuo M. Dysregulation of the Bmi-1/p16Ink4a pathway provokes an aging-associated decline of submandibular gland function. Aging Cell 2015; 14: $616-624$.

4. Feng $X$ and Laura L. Aging-related changes in quantity and quality of saliva: Where do we stand in our understanding? Journal of texture studies. Special Issue: Saliva and Food Oral Processing 2019; 50: 27-35.

5. Rizwan A. Free Radicals, Antioxidants and Diseases. Published: August 1st 2018. DOI: 10.5772/intechopen. 70075. ISBN: 978-1-78923-565-4. Print ISBN: 978-178923-564-7. Copyright year: 2018.

6. Villines Z. Antioxidants and free radicals. 2017. Available from: www.medicalnewstoday.com
7. Meija, J. Atomic weights of the elements 2013 (IUPAC Technical Report). Pure and Applied Chemistry 2016; 88: 265-91.

8. Ruyle G. Poisonous Plants on Arizona Rangelands . The University of Arizona. Archived from the original (PDF) on 2004-07-15. Retrieved 2009-01-05.

9. Son H, Lee S, Yoon R, Lee H, Lee I, Kim S, Chung W, Lee J. Effect of selenium supplementation for protection of salivary glands from iodine-131 radiation damage in patients with differentiated thyroid cancer. Hell J Nucl Med 2017; 20:62-70.

10. Helen L, Kirsten H. and David K. Current State of Knowledge on Salivary Gland Cancers. Crit Rev Oncog 2018; 23: 139-151.

11. Mehmet S. Relation of Trace Elements on Dental Health. Trace Elements - Human Health and Environment 2019; Chapter 4: p 71-84.

12. Scholzen $\mathrm{T}$ and Gerdes J: The Ki-67 protein: from the known and the unknown. In: J. Cell. Physiol 2000; 182, No. 3: pp. 311-22.

13. Nkomozepi P, Mazengenya $\mathrm{P}$ and Ihunwo A. Age-related changes in Ki-67 and DCX expression in the BALB/ c mouse brain. Int J Dev Neuro sci 2019; 72:36-47.

14. Faul F, Erdfelder E and Lang AG: G*Power 3: A flexible statistical power analysis program for the social, behavioral, and biomedical sciences. Behavior Research Methods 2007; 39: 175-191.

15. Benevenga $\mathrm{N}$, Eckhert $\mathrm{C}$ and Fahey G: Nutrient requirements of laboratory animals. The national academic press. 4 th ed. Washington D.C; 1995, pp. $11-79$.

16. Salkou S, Tsamandas A and Bonikos D: The potential role of bcl-2, box, and ki-67 expression in thymus of patients with myasthenia gravis, and their correlation with clinicopathologic parameters. Eurpean Journal of CardioThoracic Surgery 2001; 20: 712-21.

17. Luo S, Tan W and Deng W.: Expression of albumin, IGF-1, IGFBP-3 in tumor tissues and adjacent non-tumor tissues of hepatocellular carcinoma patients with cirrhosis. World J Gastroenterol 2005; 11:4272-76.

18. Cowen D, Troncoso P and Khoo V: Ki-67 Staining Is an Independent Correlate of Biochemical Failure in Prostate Cancer Treated with Radiotherapy. Clinical Cancer Research 2002; 8:1148-54. 
19. Wong N, Young R and Malcomson RI: Prognostic indicator for gastrointestinal stromal tumours: A clinicopathological immunihistochemical study of 108 resected cases of the stomach. Histopathology 2003; 43:118-26.

20. Grawish M and Zyada M: Inhibition of 4-NQO induced F433 rat tongue carcinogenesis by oleuropein-rich extract. Med Oncol. 2011; 28:1163-8.

21. Steel R, Torrie J and Dicky D: Principles and Procedures of Statistics, a Biometrical Approach. $3^{\text {rd }}$ Edition, McGraw Hill, Inc. Book Co., New York. 1997; 3:352-358.

22. Ahmadv H, Babaeenezhad E and Nayeri H: Selenium effects on antioxidant and inflammatory indices in renal ischemia-reperfusion injury in rats. J Renal Inj Prev. 2019; 8:71-77.

23. Avnika G, Shivani B and Rajesh K: Aging and Periodontium. Dental Journal of Advance Studies 2013; 01: 026-029.

24. Harsh V. and Pankaj J: Age related changes in submandibular salivary gland. International Journal of Medical and Health Research 2016; 2:15-17.

25. Blagosklonny M: Aging: ROS or TOR. Cell Cycle 2008; 7: 3344-3354.

26. Azevedo L, Damante J and Lara V: Age-related changes in human sublingual glands: a post mortem study. Arch Oral Biol 2005; 50:565-74

27. Josiane C, Elen S and Luciana R: Morphology and morphometry of the human sublingual glands in mouth floor enlargements of edentulous patients. J. Appl. Oral Sci $2013 ; 21: 6-7$

28. Ujang T: Selenium: its role as antioxidant in human health. Environ Health Prev Med 2008; 13: 102-108.

29. Ning W, Hor-Yue $T$ and Sha L: Supplementation of Micronutrient Selenium in Metabolic Diseases: Its Role as an Antioxidant. Oxidative Medicine and Cellular Longevity 2017, Article ID 7478523, 13 pages. https://doi. org/10.1155/2017/7478523.

30. Miller I, Min M and Yang C: Ki67 is a Graded Rather than a Binary Marker of Proliferation versus Quiescence. Cell Rep 2018; 24:1105-1112.

31. Tanaka A, Watanabe Y and Kato H: Immunohistochemical changes related to ageing in the mouse hippocampus and subventricular zone. Mech Ageing Dev 2007; 128:303-310

32. Shaw A, Joshi S and Greenwood H: Aging of the innate immune system. Curr. Opin. Immunol 2010; 22: 507-513.

33. Rossi D, Bryder D and Seita J: Deficiencies in DNA damage repair limit the function of haematopoietic stem cells with age. Nature 2007; 447: 725-729.

34. Sangeeta D, Ercument D and Rohit N: Inhibition of TGF-beta signaling promotes human pancreatic beta cell replication. Diabetes Publish Ahead of Print 2016; 3: 1-42.

35. Lara J, Patricia R and David B: Selenium preserves keratinocyte stemness and delays senescence by maintaining epidermal adhesion. Aging (Albany NY) 2017; 9: 2302-2315.

36. Xiaoming S, Aizhan B and Timothy M: Ki-67 contributes to normal cell cycle progression and inactive $\mathrm{X}$ heterochromatin in p21 checkpoint-proficient human cells. Molecular and Cellular Biology 2017; doi: 10.1128/ MCB.00569-16.

37. Vered A, Buchner $\mathrm{P}$ and Boldon D: Age-related histomorphometric changes in labial salivary glands with special reference to the acinar component. Experimental Gerontology 2000; 35: 1075-1084.

38. Huawei Z: Selenium as an Essential Micronutrient: Roles in Cell Cycle and Apoptosis. Molecules 2009; 14: 1263 1278 . 